\title{
FLUID MOVEMENT IN A CHANNEL OF VARYING GAP WITH PERMEABLE WALLS COVERED BY POROUS MEDIA
}

\author{
P. N. SHIVAKUMAR, $\dagger$ S. NAGARAJ, R. VEERABHADRAIAH and N. RUDRAIAH \\ UGC-DSA Centre in Fluid Mechanics, Department of Mathematics, Central College, \\ Bangalore University, Bangalore-560001, India
}

\begin{abstract}
Blood flow in arteries idealized into a channel of varying gap bounded by porous layer is studied. Analytical solutions are obtained using Beavers and Joseph slip condition by three approximate methods depending upon the geometrical configuration. The general solutions are applied to a particular problem of smooth constriction idealized into an artery with stenosis. The resistance of the porous layer to the flow in the channel and the shear stress at the nominal surface are discussed in detail. It is shown that for a given porous layer, depending on the value of the porous parameter $\alpha \sigma_{0}$, this may lead to an increase or decrease in the resistance and the shear stress may be used in evaluating the performance of various prosthetic devices which ultimately may be implanted in the living system.
\end{abstract}

\section{INTRODUCTION}

FLUID MOVEMENT in a channel of varying gap with permeable walls covered by a layer of porous material is discussed. The motivation of this investigation comes from the study of abnormal flow in the arterial system caused by the presence of occlusion or stenosis. At various locations in the arterial system, stenosis may develop (Young [1]) due to abnormal intravascular growth. It may also be due to the abnormal accumulation of fluid in the tissue bounding the artery. Arteries may also be narrowed by the development of atherosclerotic plaques which are closely connected with the blood flow through the artery bounded by a thin layer of tissue, idealized into a porous medium, which separates the blood flow from the flow of other physiological fluids (hereafter called PF). The stenosis developed in the artery causing the abnormal flow is an important factor in the development and progression of arterial diseases. Therefore, the study of movement and accumulation of PF in the tissue and their effect on blood flow in the artery is an important problem because the flow characteristics in the vicinity of the resulting protuberance may significantly be altered. Therefore, the results of the present study throw light on the understanding of the important flow characteristics in the arterial system, namely, the pressure, shear stress and possible changes in them. These in turn are related, respectively, to the physiologically important problems involving

(i) increase in resistance to the blood flow,

(ii) possible damages to the red and endothelial cells due to the existence of high shear zones, and

(iii) possible transition from a laminar to turbulent flow inside the blood vessel creating high intensity shear zones unfavourable to the blood flow and arterial wall.

The flow in a channel of varying gap bounded by rigid walls has been investigated by many authors (see Langlois [2], Young [1], Lee and Fung [3], Chow and Soda [4], Chandrasekhara and Rudraiah [5]). The results obtained from these analyses using rigid boundaries are not of much use in understanding the characteristics of flow in arteries, because they are bounded by tissues which are idealized into a porous medium where one has to use a slip condition at the bounding surface similar to the one postulated by Beavers and Joseph [6] (hereafter called BJ condition). Therefore, in this study we consider a two dimensional channel of varying gap bounded on both sides by a porous layer as shown in Fig. 1. We shall take the blood space as the channel and the tissue space as the porous layer. The blood flow in the channel is governed by the Navier-

† Permanent address: Department of Applied Mathematics, University of Manitoba, Winnipeg, Manitoba R3T 2N2, Canada. 


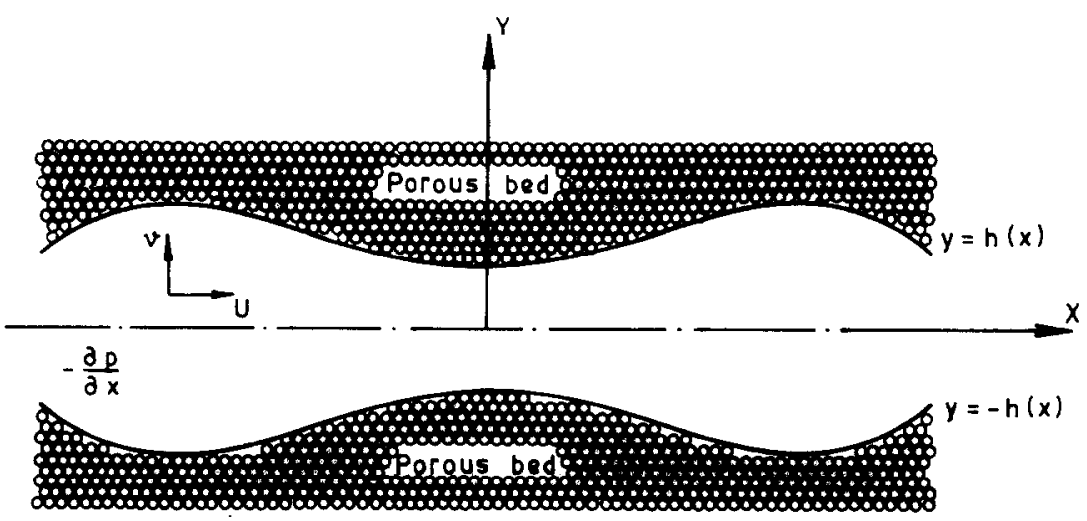

Fig. 1. Physical model.

Stokes equations and that in the porous layer is governed by the Darcy law with the BJ condition at the surface of the porous layer. In reality, direct numerical integration of these equations would be the best way to understand all the characteristics of the flow, if it is possible. However, there are many difficulties, for example, in approximating the irregular wall shape bounding the porous material while applying numerical methods. Therefore, in the present analysis we solve the system of equations analytically using the BJ slip condition with the object of understanding the physics of the problem with minimum mathematics. For this, we assume that the thickness of the porous layer is much larger than the width of the flow in the channel so that we can directly use the BJ condition at the bounding surface of the channel. To obtain analytical solutions using this BJ condition three approximate methods are developed for the three different configurations. In the first method, wall slope is assumed to be negligible and the results obtained are similar to those of Rudraiah et al. [7]. In the second method wall curvature is assumed to be negligible and the analysis is carried out by approximating the channel to that of a divergent wedge bounded by a porous layer with a source at the vertex. In the third method, the results of the second method are expanded in power series in terms of the wall slope and the results are applied to the problem of flow in a channel with constriction which is idealized into an artery with stenosis. It is shown that the resistance of the wall to the flow and the shear stress at the wall are greatly affected by the porous parameter $\bar{\alpha} \sigma_{0}$, where $\bar{\alpha}$ is the slip parameter and $\sigma_{0}=h / \sqrt{ } k, k$ is the permeability of the tissues and $h$ is the width of flow in the channel. For a given porous layer, depending on the value of $\bar{\alpha} \sigma_{0}$, this may lead to an increase or decrease in the resistance and shear stress. This information may be useful in evaluating the performance of various prosthetic devices that may ultimately be implanted in vivo (i.e. the flow of PF in their natural habit). We note that the assumption-the thickness of the porous layer is large compared to the thickness of the fluid in the channel-made in this paper to use the BJ condition may not be valid directly to the biological process explained above. Because, in reality the thickness of the intersticial space idealized into a porous medium is much smaller (of order $1 \mu \mathrm{m}$ ) than the thickness of the blood channel (usually varies in the range $0-7 \mu \mathrm{m}$ ). In that case the BJ condition cannot be used directly because it is independent of the thickness of the porous layer and ignores the variation of velocity in the porous layer. Work is in progress to include the finite thickness of the porous layer by modifying the BJ condition (Rudraiah [8]) by incorporating the variation of velocity in the porous layer. The results of the present analyses, however, are useful to solve this more general complicated problem of flow in a channel of varying gap bounded by this porous layer.

\section{FORMULATION OF THE PROBLEM}

The fluid flowing in the channel is assumed to be steady, homogeneous, incompressible and Newtonian. The coordinate system is chosen in such a way that the channel lies in the $x-y$ plane (Fig. 1) with $x$-axis coinciding with the centre line of the channel and the $y$ axis perpendicular to the $x-z$ plane. It is further assumed that the channel has symmetry 
about the $x$-axis. It is important to note that in reality complex three-dimensional flow patterns develop near the stenosis and blood behaves as a non-Newtonian fluid which are virtually impossible to analyse in full generality. Blood, a suspension in the artery, behaves as a Newtonian fluid at large shear rates and in some instances, the stenosis is known to be more "collar like" (Young [1]) with some degree of axial symmetry. Therefore, the assumptions of Newtonian fluid and axial symmetry made in this study are reasonably valid. However, the porous layer is assumed to be homogeneous, isotropic and densely packed so that the usual Darcy law is valid. The nominal surface (see Beavers and Joseph [6]) bounding the channel on either side is assumed to be represented by the curves

$$
y= \pm h(x)
$$

where $h(x)$ is continuous and positive for all $x$. The flow in the channel is governed by the Navier-Stokes equation and that in the porous layer is governed by Darcy law and the flow is coupled through $\mathrm{BJ}$ condition.

The equations of motion, neglecting inertia effects in the channel are (see Fig. 1)

$$
\begin{aligned}
\frac{\partial u}{\partial x}+\frac{\partial v}{\partial y} & =0 \\
\frac{1}{\mu} \frac{\partial p}{\partial x} & =\nabla^{2} u \\
\frac{1}{\mu} \frac{\partial p}{\partial y} & =\nabla^{2} v
\end{aligned}
$$

and those in the porous layer are governed by Darcy velocity

$$
Q_{x}=-\frac{k}{\mu} \frac{\partial p}{\partial x}, \quad Q_{y}=-\frac{k}{\mu} \frac{\partial p}{\partial y}
$$

where $\nabla^{2}=\partial^{2} / \partial x^{2}+\partial^{2} / \partial y^{2}, u$ and $v$ are the velocity components in the $x$ and $y$ directions $p$ is the pressure, $\mu$ is the viscosity.

These equations are solved using the boundary conditions

$$
\begin{aligned}
& \frac{\mathrm{d} u}{\mathrm{~d} y}=-\bar{\alpha}\left(\frac{u B_{1}-Q_{1}}{\sqrt{ } k}\right) \quad \text { at } \quad y=h(x), \\
& \frac{\mathrm{d} u}{\mathrm{~d} y}=\bar{\alpha}\left(\frac{u_{B}-Q_{1}}{\sqrt{ } k}\right) \quad \text { at } \quad y=-h(x), \\
& v=\epsilon Q_{y}, \quad Q_{1}=-\frac{k}{\mu} \frac{\partial p}{\partial x}, \\
& \int_{-h(x)}^{+h(x)} u \mathrm{~d} y=m
\end{aligned}
$$

where $m$ is the net flux through the channel, $u_{B}, u_{B_{1}}$ are the slip velocities, $k$ is the permeability of the porous material and $\bar{\alpha}$ is the dimensionless constant called slip parameter. The conditions (2.6) and (2.7) are the BJ conditions.

\section{SOLUTIONS OF THE PROBLEM}

To find the solution of the problem three approximate methods depending upon the three different physical situations are developed and these are discussed in this section.

\subsection{First approximation: Wall slope everywhere negligible}

If the wall slope $h^{\prime}(x)$ is everywhere small compared to unity, it is reasonable to assume that at each value of $x$, the components of velocity and pressure gradient are 
approximately equal to those obtained in the case of uniform channel flow. This approximation leads to the velocity distribution given by Rudraiah et al. [7] with a pressure gradient parallel to the axis of the channel. Under these approximations the basic equations are:

$$
\begin{aligned}
\frac{1}{\mu} \frac{\partial p}{\partial x} & =\frac{\partial^{2} u}{\partial y^{2}}, \\
\frac{\partial p}{\partial y} & =0, \\
v & =0 .
\end{aligned}
$$

Eliminating $p$ from these equations we get

$$
\frac{\mathrm{d}^{3} u}{\mathrm{~d} y^{3}}=0
$$

Solving (3.4) using the boundary conditions (2.6)-(2.9) we get

$$
U=\frac{m}{2 h}+\frac{\bar{\alpha} \sigma}{4 h^{3}}\left[\frac{m-2 h Q_{1}}{3+\bar{\alpha} \sigma}\right]\left(h^{2}-3 y^{2}\right)
$$

where

$$
m=\frac{2 h^{3}}{3}\left(\frac{3 \bar{\alpha}+\sigma(3+\bar{\alpha} \sigma)}{\bar{\alpha} \sigma^{2}}\right)\left(-\frac{1}{\mu} \frac{\partial p}{\partial x}\right)
$$

The expression for the pressure is

$$
\frac{p}{\mu}=\frac{3 \bar{\alpha}}{2} \int_{c}^{x} \frac{\sigma\left(2 h Q_{1}-m\right)}{(3+\alpha \sigma) h^{3}} \mathrm{~d} x
$$

where $c$ is a constant of integration. Equations (3.5) and (3.7) in the limit of $\sigma \rightarrow \infty$ tends to

$$
\begin{aligned}
u & =\frac{3 m}{4 h}\left(1-\frac{y^{2}}{h^{2}}\right), \\
\frac{1}{\mu} \frac{\partial p}{\partial x} & =-\frac{3}{2} \frac{m}{h^{3}}
\end{aligned}
$$

which are the usual solutions for a channel flow bounded by rigid impermeable walls.

Equations (3.3) and (3.5) satisfy the boundary conditions exactly. The components of velocity given by (3.3) and (3.5) are the possible components if and only if

$$
\begin{aligned}
\left|h^{\prime}(x)\right| & \ll 1, \\
\left|h(x) h^{\prime}(x)\right| & \ll 1, \\
\left|h^{2}(x) h^{\prime}(x)\right| & \ll 1 .
\end{aligned}
$$

The conditions (3.10) imply that solutions (3.5)-(3.7) are valid only when the wall slope is small.

\subsection{Second approximation: Wall curvature everywhere negligible}

The restriction made above, viz. that the wall slope is negligible, (i.e. $\left.h^{\prime}(x) \ll 1\right)$ can be relaxed by assuming the flow locally to be as if $h(x)$ were a linear function of $x$. Depending on the sign of $h^{\prime}(x)$ we have different geometrical situations of the channel. If $h^{\prime}(x)$ is positive, the channel is approximated by a divergent wedge with a source of flux 
at its vertex. If $h^{\prime}(x)$ is negative, the wedge is convergent with a sink at its vertex. The analysis is carried out assuming $h^{\prime}(x)$ as positive and similar results can be obtained when $h^{\prime}(x)$ is negative.

In this case the equations of motion (2.2)-(2.4) are written in cylindrical coordinates $(r, \theta)$ in the form

$$
\begin{gathered}
\frac{\partial}{\partial r}(u r)+\frac{\partial v}{\partial \theta}=0 \\
\frac{1}{\mu} \frac{\partial p}{\partial r}=\nabla^{2} u-\frac{u}{r^{2}}-\frac{2}{r^{2}} \frac{\partial v}{\partial \theta} \\
\frac{1}{\mu} \frac{\partial p}{\partial \theta}=r\left[\nabla^{2} v-\frac{v}{r^{2}}+\frac{2}{r^{2}} \frac{\partial u}{\partial \theta}\right]
\end{gathered}
$$

where $u, v$ are the components of velocity in the radial and azimuthal directions, respectively, and

$$
\nabla^{2}=\frac{\partial^{2}}{\partial r^{2}}+\frac{1}{r} \frac{\partial}{\partial r}+\frac{1}{r^{2}} \frac{\partial^{2}}{\partial \theta^{2}}
$$

We assume that the radial component of velocity to be of the form

$$
u=\frac{f(\theta)}{r},
$$

then it follows from the equation of continuity that

$$
\frac{\partial v}{\partial \theta}=0
$$

To satisfy the continuity of the normal component of velocity we assume that $v=0$ because the porous medium is assumed to be saturated. Then the above equations of motion become

$$
\begin{aligned}
& \frac{1}{\mu} \frac{\partial p}{\partial r}=\frac{f^{\prime \prime}(\theta)}{r^{3}} \\
& \frac{1}{\mu} \frac{\partial p}{\partial \theta}=\frac{2}{r^{2}} f^{\prime}(\theta)
\end{aligned}
$$

Eliminating $p$ between (3.15) and (3.16) we get

$$
f^{\prime \prime \prime}(\theta)+4 f^{\prime}(\theta)=0 \text {. }
$$

The boundary conditions (2.6)-(2.9) now take the form

$$
\begin{aligned}
& f(\alpha) \sin \alpha+f^{\prime}(\alpha) \cos \alpha=-\bar{\alpha} \sigma_{0}(f(\alpha)-Q) \quad \text { when } \quad \theta=\alpha, \\
& -f(-\alpha) \sin \alpha+f^{\prime}(-\alpha) \cos \alpha=\bar{\alpha} \sigma_{0}(f(-\alpha)-Q) \quad \text { when } \quad \theta=-\alpha \text {, } \\
& \int_{-\alpha}^{+\alpha} f(\theta) \mathrm{d} \theta=m
\end{aligned}
$$

where

$$
\begin{aligned}
& Q=Q_{1} R_{0}, \quad R_{0}=\frac{r_{0}}{\cos \alpha}, \quad Q_{1}=-\frac{k}{\mu} \frac{\partial p}{\partial r}, \\
& \left.\sigma_{0}=\frac{R_{0}}{\sqrt{ } k} \quad \text { (i.e. } h \sigma_{0}=R_{0} \sigma\right)
\end{aligned}
$$


$r_{0}$ is the value of $r$ at $\theta=0$. Solving (3.17) using the boundary conditions (3.18) to (3.20) we obtain

$$
\begin{aligned}
f(\theta) & =2 m \gamma\left(\sin ^{2} \alpha-\sin ^{2} \theta\right)+4 \lambda \gamma \cos ^{2} \theta+K_{1}, \\
u & =\frac{2 m \gamma}{r}\left(\sin ^{2} \alpha-\sin ^{2} \theta\right)+\frac{4 \lambda \gamma \cos ^{2} \theta}{r}+\frac{K_{1}}{r}, \\
v & =0, \\
\frac{1}{\mu} \frac{\partial p}{\partial r} & =-\frac{\gamma(m+2 \lambda) \cos 2 \theta}{r^{3}}, \\
\frac{1}{\mu} \frac{\partial p}{\partial \theta} & =-\frac{4 \gamma(m+2 \lambda) \sin 2 \theta}{r^{2}}, \\
\frac{p}{\mu} & =\frac{2 \gamma(m+2 \lambda) \cos 2 \theta}{r^{2}}+\text { constant }
\end{aligned}
$$

where

$$
\begin{aligned}
\gamma & =\frac{1}{\sin 2 \alpha-2 \alpha \cos 2 \alpha+\frac{4 \alpha \cos \alpha \sin 2 \alpha}{\sin \alpha+\bar{\alpha} \sigma_{0}}}, \\
\lambda & =-\frac{\alpha \bar{\alpha} \sigma_{0} Q}{\sin \alpha+\bar{\alpha} \sigma_{0}}, \\
K_{1} & =\gamma\left[\frac{2 m \cos \alpha \sin 2 \alpha+\bar{\alpha} \sigma_{0} Q(\sin 2 \alpha+2 \alpha)}{\sin \alpha+\bar{\alpha} \sigma_{0}}\right] .
\end{aligned}
$$

We note that (3.22), (3.23) and (3.26) in the limit $\sigma_{0} \rightarrow \infty$ tend to

$$
\begin{aligned}
u & =\frac{m}{r} \frac{\sin ^{2} \alpha-\sin ^{2} \theta}{\sin \alpha \cos \alpha-\alpha+2 \alpha \sin ^{2} \alpha}, \\
v & =0, \\
\frac{p}{\mu} & =\frac{m}{r^{2}} \frac{\cos ^{2} \theta-\sin ^{2} \theta}{\sin \alpha \cos \alpha-\alpha+2 \alpha \sin ^{2} \alpha}+\text { constant }
\end{aligned}
$$

which are exactly the same as those given by Longlois [2] in the case of a channel bounded by rigid boundaries.

Prior to applying these results to the problem of flow through a channel of varying gap bounded by a porous layer, we transform them to cartesian coordinates, using the transformation (Fig. 2):

$$
\begin{aligned}
u_{x} & =u \cos \theta, & u_{y}=u \sin \theta \\
\cos \theta & =(x-\mathbf{X}) / r, & \sin \theta=y / r \\
\sin \alpha & =\frac{D}{\sqrt{ } 1+D^{2}}, & \cos \alpha=\frac{1}{\sqrt{ } 1+D^{2}}
\end{aligned}
$$

where

$$
\alpha=\arctan D, \quad r=\sqrt{(x-\mathbf{X})^{2}+y^{2}},
$$

$x, y$ are the nondimensional coordinates. The components of pressure gradient are given by 


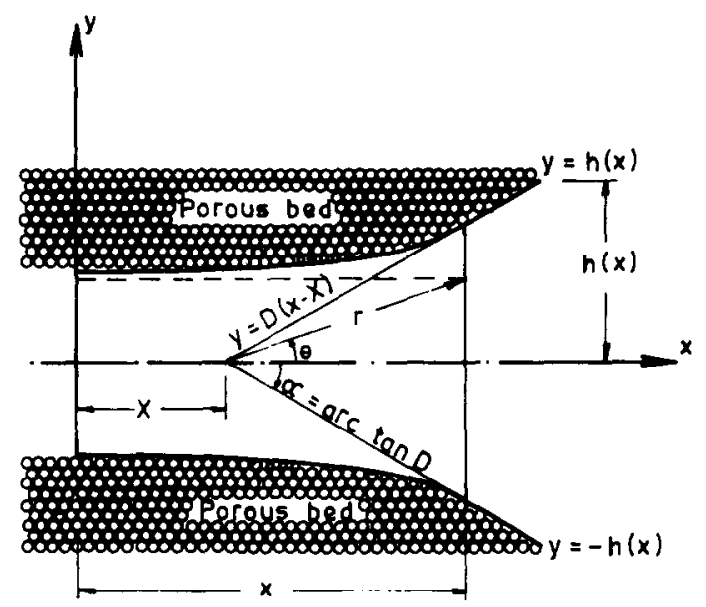

Fig. 2. Flow geometry.

$$
\begin{aligned}
& \frac{\partial p}{\partial x}=\frac{x-\mathbf{X}}{r} \frac{\partial p}{\partial r}-\frac{y}{r^{2}} \frac{\partial p}{\partial \theta} \\
& \frac{\partial p}{\partial y}=\frac{y}{r} \frac{\partial p}{\partial r}+\frac{x-\mathbf{X}}{r^{2}} \frac{\partial p}{\partial \theta}
\end{aligned}
$$

Equations (3.22)-(3.26), using (3.30), become

$$
\begin{gathered}
u_{x}=\frac{m A_{0} D^{3} h\left(h^{2}-y^{2}\right)}{\left(h^{2}+D^{2} y^{2}\right)^{2}}+\frac{D h}{\left(h^{2}+D^{2} y^{2}\right)^{2}}\left[2 A_{0} B_{0}\left(1+D^{2}\right)^{3 / 2} h^{2}+C_{0}\left(h^{2}+D^{2} y^{2}\right)\right], \\
u_{y}=\frac{m A_{0} D^{4}\left(h^{2}-y^{2}\right) y}{\left(h^{2}+D^{2} y^{2}\right)^{2}}+\frac{D^{2} y}{\left(h^{2}+D^{2} y^{2}\right)^{2}}\left[2 A_{0} B_{0}\left(1+D^{2}\right)^{3 / 2} h^{2}+C_{0}\left(h^{2}+D^{2} y^{2}\right)\right], \\
\frac{1}{\mu} \frac{\partial p}{\partial x}=-2 A_{0}\left(1+2 \frac{B_{0}}{m} \sqrt{ }+D^{2}\right) m D^{3}\left(1+D^{2}\right) h\left[\frac{h^{2}-3 D^{2} y^{2}}{\left(h^{2}+D^{2} y^{2}\right)^{3}}\right], \\
\frac{1}{\mu} \frac{\partial p}{\partial y}=-2 A_{0}\left(1+2 \frac{B_{0}}{m} \sqrt{ } 1+D^{2}\right) m D^{4}\left(1+D^{2}\right) y\left[\frac{3 h^{2}-D^{2} y^{2}}{\left(h^{2}+D^{2} y^{2}\right)^{3}}\right]
\end{gathered}
$$

where

$$
\begin{aligned}
A_{0} & =\frac{1}{E+\left[4 \alpha D /\left(D+\bar{\alpha} \sigma_{0} \sqrt{\left.1+D^{2}\right)}\right]\right.}, \\
\frac{B_{0}}{m} & =-\frac{\alpha \bar{\alpha} \sigma_{0} Q_{0}}{D+\bar{\alpha} \sigma_{0} \sqrt{1+D^{2}}}, \\
\frac{C_{0}}{m} & =A_{0}\left[\frac{2 D+\bar{\alpha} \sigma_{0} Q_{0}\left\{D+\alpha\left(1+D^{2}\right)\right\} \sqrt{1+D^{2}}}{D+\bar{\alpha} \sigma_{0} \sqrt{1+D^{2}}}\right], \\
E & =D-\left(1-D^{2}\right) \arctan D, \\
h & =D(x-\mathbf{X}), \quad Q_{0}=\frac{Q_{1}}{m} .
\end{aligned}
$$

If the curvature of the walls is everywhere small [i.e. $\left.h(x) h^{\prime \prime}(x) \ll 1\right)$ then at each value of $x$ the flow in the channel of varying gap may be approximated to a flow in a wedge with vertex at $[\mathbf{X}(x), 0]$ and vertex angle $[2 \arctan D(x)]$, where 


$$
\begin{aligned}
& D(x)=h^{\prime}(x), \\
& \mathbf{X}(x)=x-\frac{h(x)}{D(x)} .
\end{aligned}
$$

The velocity components given by (3.31) and (3.32) satisfy the boundary conditions and (3.33) and (3.34) satisfy the differential equations approximately provided as

$$
\begin{aligned}
\left|h(x) h^{\prime \prime}(x)\right| & \ll 1, \\
\left|h^{2}(x) h^{\prime \prime}(x)\right| & \ll 1
\end{aligned}
$$

for all $x$.

If (3.36) is satisfied, then it is very easy to verify that

$$
\mathrm{d} p=\frac{\partial p}{\partial x} \mathrm{~d} x+\frac{\partial p}{\partial y} \mathrm{~d} y
$$

and we have

$$
\begin{aligned}
\frac{p}{\mu}=2 m \int_{x}^{c} \frac{A_{0}\left(1+\left(B_{0} / m\right) \sqrt{ } 1+D^{2}\right)\left(1+D^{2}\right) D^{3}}{h^{3}} \mathrm{~d} x \\
-m A_{0}\left(1+\frac{B_{0}}{m} \sqrt{ } 1+D^{2}\right) D^{4}\left(1+D^{2}\right)\left(\frac{y}{h}\right)^{2}\left[\frac{3 h^{2}+D^{2} y^{2}}{\left(h^{2}+D^{2} y^{2}\right)^{2}}\right]
\end{aligned}
$$

where $c$ is a constant of integration. Equations (3.31) to (3.34) and (3.37) in the limit of $\sigma_{0} \rightarrow \infty$ tend to

$$
\begin{gathered}
u_{x}=\frac{m D^{3} h}{E} \frac{h^{2}-y^{2}}{\left(h^{2}+D^{2} y^{2}\right)^{2}}, \\
u_{y}=\frac{m D^{4} y}{E} \frac{h^{2}-y^{2}}{\left(h^{2}+D^{2} y^{2}\right)^{2}}, \\
\frac{1}{\mu} \frac{\partial p}{\partial x}=-\frac{2 m\left(1+D^{2}\right) D^{3} h}{E}\left[\frac{h^{2}-3 D^{2} y^{2}}{\left(h^{2}+D^{2} y^{2}\right)^{3}}\right], \\
\frac{1}{\mu} \frac{\partial p}{\partial y}=-\frac{2 m\left(1+D^{2}\right) D^{4} y}{E}\left[\frac{h^{2}-3 D^{2} y^{2}}{\left(h^{2}+D^{2} y^{2}\right)^{3}}\right], \\
\frac{p}{\mu}=2 m \int_{x}^{c} \frac{\left(1+D^{2}\right) D^{3}}{E h^{3}} \mathrm{~d} x-\frac{m}{E} D^{4}\left(1+D^{2}\right)\left(\frac{y}{h}\right)^{2}\left[\frac{3 h^{2}+D^{2} y^{2}}{\left(h^{2}+D^{2} y^{2}\right)^{2}}\right]
\end{gathered}
$$

which coincide with those given by Langlois [2] in the case of a channel bounded by rigid boundaries.

3.3. Third approximation method: Power series expansion in the wall slope

The second method discussed above leads to rather cumbersome results even for analytically simple form of $h(x)$ and also it may happen that the function $h(x)$ satisfying (3.36) is such that $h^{\prime}(x)$ is small but not negligible. Then the modified condition

$$
\left|D^{n}\right|=\left|h^{\prime}(x)^{n}\right| \ll 1
$$

is satisfied for some positive integer $n$. Now for $n>1$ the results of the second method are expanded in power series in $D$ and the terms of the $n$th or higher order in $D$ are 
neglected. For simplicity, we restrict our analyses to the case when $n=3$. Expanding arc $\tan D$ in power series of $D$, we have

$$
\arctan D=D-\frac{1}{3} D^{3}+\frac{1}{5} D^{5}+O\left(D^{7}\right) .
$$

The function $E$, expanded in terms of $D$, is given by

$$
E=\frac{4}{3} D^{3}\left[1-\frac{2}{5} D^{2}+O\left(D^{4}\right)+\cdots\right] .
$$

Hence,

$$
\frac{D^{3}}{E}=\frac{3}{4}\left[1+\frac{2}{5} D^{2}+O\left(D^{4}\right)+\cdots\right]
$$

The expressions for the velocity components and pressure, after neglecting the third and higher order in $D$, take the form

$$
\begin{aligned}
& u_{x}=\frac{m}{h} A_{0} D^{3}\left(1-\frac{y^{2}}{h^{2}}\right)\left(1-2 D^{2} \frac{y^{2}}{h^{2}}\right) \\
& +\frac{1}{\bar{\alpha} \sigma_{0}} \frac{2 m}{h} A_{0} D^{2}\left[1-\frac{1}{2} D^{2}+\frac{D^{2}}{\left(\bar{\alpha} \sigma_{0}\right)^{2}}-2 D^{2} \frac{y^{2}}{h^{2}}-\frac{D}{\bar{\alpha} \sigma_{0}}\right], \\
& u_{y}=\frac{m}{h} A_{0} D^{4}\left(1-\frac{y^{2}}{h^{2}}\right)\left(1-2 D^{2} \frac{y^{2}}{h^{2}}\right)\left(\frac{y}{h}\right) \\
& +\frac{2 m A_{0} D^{3}}{\bar{\alpha} \sigma_{0}}\left(\frac{y}{h}\right)\left[1-\frac{1}{2} D^{2}-\frac{D}{\bar{\alpha} \sigma_{0}}+\frac{D^{2}}{\left(\bar{\alpha} \sigma_{0}\right)^{2}}-2 D^{2} \frac{y^{2}}{h^{2}}\right], \\
& \left.\frac{1}{\mu} \frac{\partial p}{\partial x}=-\frac{3 m}{2 h^{3}}\left[1-6 D^{2} \frac{y^{2}}{h^{2}}+\frac{7}{5} D^{2}\right)-2 Q_{0} D\left(1-\frac{D}{\bar{\alpha} \sigma_{0}}\right)\right], \\
& \frac{1}{\mu} \frac{\partial p}{\partial y}=-\frac{9 m D}{2 h^{3}}\left(\frac{y}{h}\right)\left(1-2 Q_{0} D\right), \\
& \frac{p}{\mu}=2 m \int_{x}^{c} \frac{A_{0} D^{3}\left(1+\left(B_{0} / m\right) \sqrt{ } 1+D^{2}\right)\left(1+D^{2}\right)}{h^{3}} \mathrm{~d} x \\
& -3 m D \frac{A_{0} D^{3}\left(1+\left(B_{0} / m\right) \sqrt{ } 1+D^{2}\right)\left(1+D^{2}\right)}{h^{2}}\left(\frac{y}{h}\right)^{2} .
\end{aligned}
$$

Equations (3.47)-(3.51) in the limit of $\sigma_{0} \rightarrow \infty$ tend to

$$
\begin{aligned}
u_{x} & =\frac{3 m}{4 h}\left(1-\frac{y^{2}}{h^{2}}\right)\left(1-2 D^{2} \frac{y^{2}}{h^{2}}+\frac{2}{5} D^{2}\right), \\
u_{y} & =\frac{3 m}{4 h} D\left(1-\frac{y^{2}}{h^{2}}\right)\left(\frac{y}{h}\right), \\
\frac{1}{\mu} \frac{\partial p}{\partial x} & =-\frac{3 m}{2 h^{3}}\left(1-6 D^{2} \frac{y^{2}}{h^{2}}+\frac{7}{5} D^{2}\right), \\
\frac{1}{\mu} \frac{\partial p}{\partial y} & =-\frac{9 m D}{2 h^{3}}\left(\frac{y}{h}\right),
\end{aligned}
$$




$$
\frac{p}{\mu}=\frac{3 m}{4}\left[2 \int_{x}^{c} \frac{1+(7 / 5) D^{2}}{h^{3}} \mathrm{~d} x-\frac{3 D y^{2}}{h^{4}}\right]
$$

which are exactly the same as those given by Langlois [2] in the absence of a porous layer.

\subsection{Comparison of the three methods}

The average pressure gradient across the channel is

$$
\Delta x=-\frac{1}{h(x)} \int_{0}^{h(x)} \frac{\partial p}{\partial x} \mathrm{~d} y
$$

$\Delta x_{i}$ is calculated in the neighbourhood of a given value of $x$ in each of the method discussed above and the weight functions $F_{i}(D)$ are compared where $F_{i}(D)$ are given by

$$
\Delta x_{i}=\frac{3 m}{2 R h^{3}} F_{i}(D), \quad i=1,2,3 .
$$

Here $F_{i}(D)(i=1,2,3)$ depend upon the method used and are given by

$$
\begin{aligned}
& F_{1}(D)=\frac{1-2 Q_{0}}{1+\left(3 \sigma_{1} / \bar{\alpha} \sigma_{0}\right)}, \\
& F_{2}(D)=\frac{4 A_{0} D^{3}}{3\left(1+D^{2}\right)}\left[1+\frac{2 B_{0}}{m} \sqrt{ } 1+D^{2}\right], \\
& F_{3}(D)=\left(1-\frac{3 D^{2}}{5}\right)-2 Q_{0} D\left(1-\frac{D}{\bar{\alpha} \sigma_{0}}\right) .
\end{aligned}
$$

These weight functions are numerically evaluated for different values of $\bar{\alpha} \sigma_{0}$, for a particular value of $Q_{0}$ and are compared in Fig. 3. We see that $F_{i}(D)$ are influenced by the porous parameter $\bar{\alpha} \sigma_{0}$, and have values smaller than those in the absence of a porous layer.

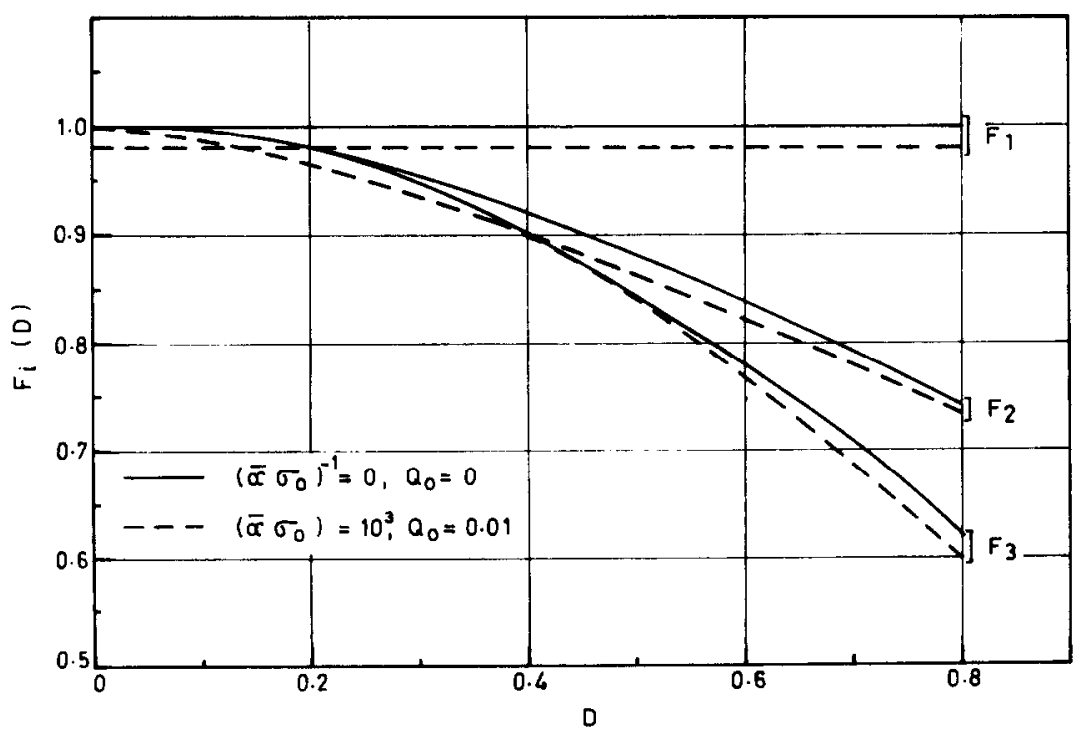

Fig. 3. Comparison of weight functions for $Q_{0}=0.01$. 


\section{FLOW THROUGH A CHANNEL WITH A SMOOTH CONSTRICTION}

The above theory is applied to the problem of flow through a channel with a smooth axisymmetric constriction (Fig. 4) defined in nondimensional variables, by

$$
h(x)=H_{0}-\frac{\delta m}{2}\left(1+\cos \frac{\pi x}{x_{0}}\right)
$$

where $\delta m$ is the maximum projection of the constriction and $H_{0}$ is the half-width of the channel. This is an idealization into an artery with stenosis.

The third approximation is valid when the condition (3.43) is satisfied, viz.

$$
\left|D^{n}\right|=\left|h^{\prime}(x)^{n}\right| \ll 1
$$

for some positive integer $n$.

The expression for $D$, from (4.1), is

$$
D=h^{\prime}(x)=\frac{\pi \delta m}{2 x_{0}} \sin \frac{\pi x}{x_{0}} .
$$

We note that the condition $\left|D^{n}\right| \ll 1$ will be satisfied if the following nondimensional quantities take the values

$$
L=4.0, \quad x_{0}=1.0, \quad \delta m=0.32 H_{0}, \quad H_{0}=1.0 .
$$

For these values, $|D|$ has a maximum value of 0.5 at $x=x_{0} / 2$ and satisfies the condition (3.43) for all positive values of $n$.

To determine the effects of slip and the porous layer on the flow characteristics of the stenosis, it is essential to determine the resistance to flow and the shear stress at the nominal surface. The resistance to a flow denoted by RF is defined as

$$
\mathrm{RF}=\frac{\text { average pressure drop across the channel }}{\text { momentum flux in the direction of flow }}
$$

To determine RF the average pressure drop across the channel and the flux in the direction of flow are to be known. The nondimensional average pressure drop across the channel is calculated from the expression

$$
p_{0}-p=\frac{1}{2 h L} \int_{-m(x)}^{+h(x)} \int_{0}^{L} \frac{\partial p}{\partial x} \mathrm{~d} x \mathrm{~d} y
$$

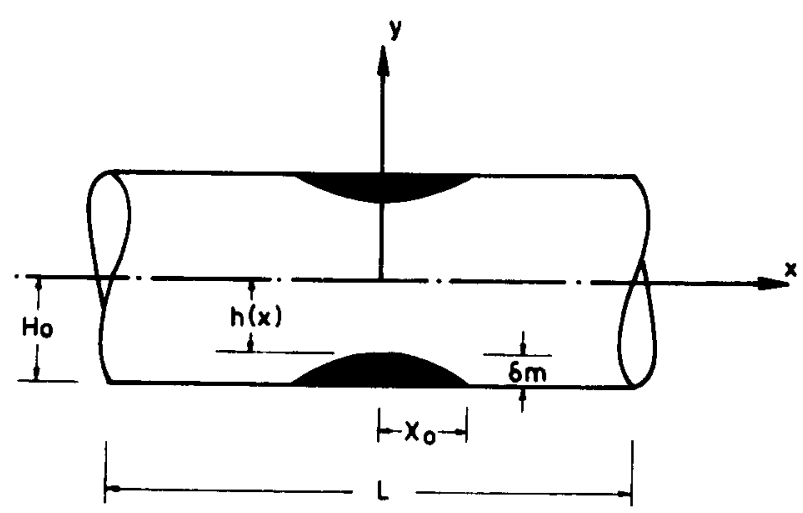

Fig. 4. Idealized geometry for stenosis. 
Using (3.49) and integrating over the interval $-h$ to $+h$ (4.4) takes the form

$$
p=\int_{0}^{L} \frac{3}{2 h L} \frac{\left[2\left(1-(3 / 5) D^{2}\right)-4 Q_{0} D\left(1-\left(D / \bar{\alpha} \sigma_{0}\right)\right)\right]}{h^{2}} \mathrm{~d} x
$$

Equation (4.5) can be written in the form

$$
p=\frac{3}{h L}\left[I_{1}+I_{2}\right]
$$

where

$$
\begin{aligned}
& I_{1}=\int_{0}^{x_{0} / 2} \frac{2\left(1-(3 / 5) D^{2}\right)-4 Q_{0} D\left(1-\left(D / \bar{\alpha} \sigma_{0}\right)\right.}{h^{2}} \mathrm{~d} x, \\
& I_{2}=\int_{x_{0} / 2}^{L / 2} \frac{2}{H_{0}^{2}} \mathrm{~d} x .
\end{aligned}
$$

The nondimensional momentum flux in the horizontal direction has the form

$$
M=\int_{-h}^{+h} u_{x}^{2} \mathrm{~d} y
$$

and after performing the indicated integration, simplifies to

$$
M=\frac{2 m^{2} K}{h}
$$

where

$$
\begin{aligned}
& K=A_{0}^{2} D^{4}\left[A_{1}+\frac{A_{2}}{3}+\frac{A_{3}}{5}+\frac{A_{4}}{7}+\frac{A_{5}}{9}\right], \\
& A_{1}=\left(D+\frac{2 q}{\bar{\alpha} \sigma_{0}}\right)^{2}, \\
& A_{2}=-2 D\left(D+\frac{2 q}{\bar{\alpha} \sigma_{0}}\right)\left(1+2 D^{2}+\frac{4 D}{\bar{\alpha} \sigma_{0}}\right), \\
& A_{3}=D^{2}\left\{4 D\left(D+\frac{2 q}{\bar{\alpha} \sigma_{0}}\right)+\left(1+2 D^{2}+\frac{4 D}{\bar{\alpha} \sigma_{0}}\right)^{2}\right\}, \\
& A_{4}=-2 D^{4}\left(1+2 D^{2}+\frac{4 D}{\bar{\alpha} \sigma_{0}}\right), \\
& A_{5}=2 D^{6}, \\
& q=1-\frac{1}{2} D^{2}-\frac{D}{\bar{\alpha} \sigma_{0}}+\frac{D^{2}}{\left(\bar{\alpha} \sigma_{0}\right)^{2}} .
\end{aligned}
$$

The expression for RF using (4.6) and (4.8) now becomes

$$
\mathrm{RF}=\frac{3}{2} \frac{I_{1}+I_{2}}{m^{2} K L}
$$

Analytical evaluation of the integral $I_{1}$ is complicated and hence it is evaluated numerically. $\mathrm{RF}$ was calculated for different values of $\bar{\alpha} \sigma_{0}$ and the behaviour of RF with $\bar{\alpha} \sigma_{0}$ is presented in Fig. 5. From this it is clear that RF is maximum for small values of $\bar{\alpha} \sigma_{0}$ 


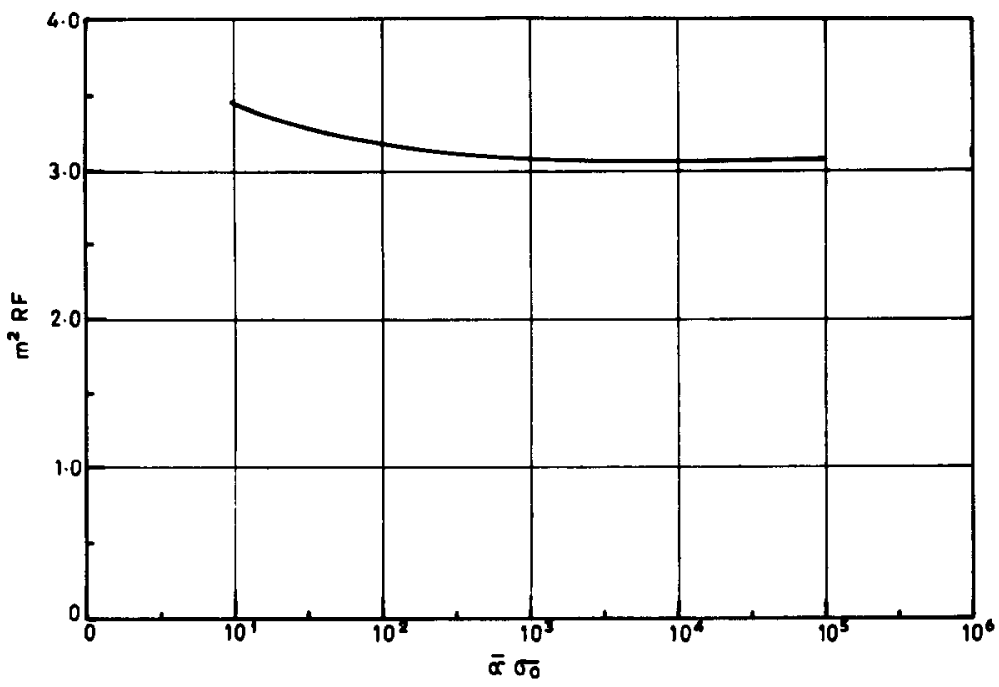

Fig. 5. Resistance force vs permeability.

( $\leq 10)$ and decreases slightly in the range $10<\bar{\alpha} \sigma_{0}<10^{3}$ because of the existence of a thin boundary layer (see Rudraiah and Masuoka [9]) and for values of $\bar{\alpha} \sigma_{0}>10^{3}, \mathrm{RF}$ remains uniform because of the negligible effect of the porous layer.

The resistance given by (4.10) is computed using the momentum flux. However in biomechanical problems, for example, in the study of blood rheology in arterial flows, the resistance to flow is normally defined (Lightfoot [10]) as

$$
\mathrm{RF}=\frac{\text { pressure drop }}{\text { volumetric flow rate }}=\frac{3\left(I_{1}+I_{2}\right)}{m h L}
$$

The resistance to flow given by (4.11) is numerically computed for different values of $\bar{\alpha} \sigma_{0}$ and the results are compared with those of (4.10). We observe that the overall nature of the resistance to flow with the porous layer is the same whether we use (4.10) or (4.11) except for a slight change.

The nondimensional shear stress at the nominal surface is next calculated using the expression

$\left(\tau_{x y}\right)_{y=h}=\frac{1}{R}\left[\frac{\partial u_{x}}{\partial y}+\frac{\partial u_{y}}{\partial x}\right]_{y=h}=\frac{2 m A_{0} D^{3}}{R h^{2}}\left[1-3 D^{2}+2 D^{4}+\frac{2 D}{\bar{\alpha} \sigma_{0}}\left(3-\frac{9 D}{2}-\frac{D}{\bar{\alpha} \sigma_{0}}\right)\right]$

for different values of $\bar{\alpha} \sigma_{0}$, and the results are presented in Table 1 .

It is seen that as expected from physical consideration that for small values of $\bar{\alpha} \sigma_{0}$ the shear stress is small which increases with increasing $\bar{\alpha} \sigma_{0}$. Larger values of $\bar{\alpha} \sigma_{0}\left(>10^{4}\right)$ correspond to the rigid case where shear stress is large.

Table $1, D=0.5, x=x_{0} / 2$

\begin{tabular}{lll}
\hline $\bar{\alpha} \sigma_{0}$ & $h$ & \multicolumn{1}{c}{$\tau_{x y}$} \\
\hline 10 & 0.84 & 0.687333 \\
$10^{2}$ & 0.84 & 0.8562346 \\
$10^{3}$ & 0.84 & 0.8826627 \\
$10^{4}$ & 0.84 & 0.8854583 \\
$10^{5}$ & 0.84 & 0.8857394 \\
\hline
\end{tabular}

When $D=0, h=0.68, \tau_{x y}=3.2439$. 
The principle parameter governing the momentum of fluid in the artery is $\bar{\alpha} \sigma_{0}$. To understand the effect of this parameter three approximate methods have been presented. These methods provide an alternate approach to the conventional method of solving two dimensional problem by conformal mapping. The weight functions $F_{i}$ are computed in each of the methods for different values of $\bar{\alpha} \sigma_{0}$ and the results are compared in Fig. 3 with those in the absence of a porous layer. From this it is clear that the weight function $F_{1}(D)$ is independent of $\bar{\alpha} \sigma_{0}$ while $F_{2}(D)$ and $F_{3}(D)$ are influenced by it and have values smaller than those in the absence of a porous layer. To study the effect of intersticial space (idealized as a porous layer) on stenosis an idealized stenosis geometry is considered and the flow characteristics such as resistance to flow and shear stress at the surface are determined. The effect of porous parameter $\bar{\alpha} \sigma_{0}$ on RF is depicted in Fig. 5. It is seen that the porous parameter $\bar{\alpha} \sigma_{0}$ greatly influences RF which decreases upto the value of $\bar{\alpha} \sigma_{0} \leq 10^{3}$ and it becomes independent of the porous parameter for values of $\bar{\alpha} \sigma_{0}$ greater than $10^{3}$. According to Rudraiah and Masuoka [9], the transition from the Darcy model to the Brinkman model takes place for values of $\bar{\alpha} \sigma_{0}$ in the range $10<\bar{\alpha} \sigma_{0}<10^{3}$. Therefore the decrease in RF in this range can be attributed to the existence of a thin boundary layer. RF remains uniform for $\bar{\alpha} \sigma_{0}>10^{3}$ because the nominal surface acts almost as a rigid impermeable wall. From Table 1 it is clear that the shear stress is small for small values of $\bar{\alpha} \sigma_{0}$ because of the boundary layer nature of Brinkman model for sparsely packed tissues. For large values of $\bar{\alpha} \sigma_{0}$ the shear stress increases as expected from physical grounds. This information, an increase or decrease of RF and shear stress with the porous parameter, is useful in evaluating the performance of various prosthetic devices that ultimately may be implanted into the living system. The work is in progress to consider the finite thickness of the porous layer using the modified BJ condition (Rudraiah [8]).

Acknowledgement-This work was partly supported by the UGC under DSA programme and by the National Research Council of Canada under grant NRC-A-8783.

\section{REFERENCES}

[1] D. F. YOUNG, J. Engng Ind. (Trans. ASME) 90, 248 (1968).

[2] W. F. LANGLOIS, Creeping viscous flow through a two dimensional channel of varying gap. Proc. Third U.S. Nat. Cong. Appl. Mech, p. 777.

[3] J.-S. LEE, and Y.-C. FUNG, J. Appl. Mech. 37, 9-16 (1970)

[4] J. C. F. CHOW and K. SODA, Phys. Fluid 15, 1700 (1972).

[5] B. C. CHANDRASEKHARA and N. RUDRAIAH, Indian J. Pure Appl. Math. 11(8), 1105-1123 (1980).

[6] G. S. BEAVERS and D. D. JOSEPH, J. Fluid Mech. 30, 197-207 (1967).

[7] N. RUDRAIAH, P. N. SHIVAKUMAR, R. VEERABHADRAIAH and D. PAL, Int. J. Math. Math. Sci., Accepted for publication (1985).

[8] N. RUDRAIAH, J. Fluld Engng (ASME), Accepted for publication (1985).

[9] N. RUDRAIAH and T. MASUOKA, Int. J. Engng Sci. 20, 27-39 (1982).

[10] E. N. LIGHTFOOT, Jr., Transport Phenomena and Living Systems, p. 17. John Wiley and Sons, New York (1974). 